\title{
RESONANT SPREAD WAVE FUNCTION IN PARABOLIC POTENTIAL
}

\section{A.S. Mazmanishvili \\ National Science Center “Kharkov Institute of Physics and Technology”, Kharkiv, Ukraine}

In this paper, we consider the parabolic potential, which as a whole is subject to dipole or quadrupole action (parametric resonance), which periodically changes with time, and the dynamics of the wave function of a particle. Based on the solutions found for the nonstationary Schrödinger equation, algorithms for calculating the dynamics of the wave function are constructed. The evolution of the wave function of a particle is analyzed. Asymptotic solutions of the equation of motion are given, using which the main characteristics of the wave packet are obtained. For selected types of potential perturbations, examples of the evolution of the wave function are given.

PACS: 28.52

\section{INTRODUCTION}

In NSC KIPT, work is underway to investigate the influence of various types of irradiation on the properties of condensed media. The mechanism of changing the properties of materials during irradiation is associated with the excitation of nonlinear localized oscillations in the lattice $[1,2]$. These oscillations in turn affect the dynamics of particles in it. The structure and arrangement of atoms in a single crystal significantly affect the character of the motion of particles in a lattice [3, 4]. This paper presents the results of the study of the resonant energy buildup, which reveal at the quantum mechanical level the physical essence of the arising processes.

We consider the quantum mechanical problem of the motion of a particle in a quadratic potential, which is subject to a dipole or quadrupole effect, which varies periodically with time. A similar formulation arises in problems when a perturbation is a trajectory of a onedimensional or two-dimensional process that modulates the potential change during the motion of a particle. A similar situation is realized, in particular, when an electron moves along the crystalline axis $[1,2]$. In this case, the role of time in the problem is played by the depth of particle penetration, and the function of perturbation, describes the forced oscillations of the crystal lattice. Another important example is related to the calculation of the rate of chemical reaction near the localized anharmonic vibrations of atoms caused by thermal fluctuations or external influence $[3,4]$. In this case, due to the large amplitude of localized anharmonic vibrations, the position of the potential well in which the particle is located can no longer be considered fixed, which requires a review of the problem of calculating the wave function taking into account the dynamics of the potential well.

The paper will consider the time evolution of a particle with the initial wave function

$$
\psi\left(x_{0}, 0\right)=\left(\frac{m \omega}{\pi \hbar}\right)^{1 / 4} \exp \left(-\frac{m \omega}{2 \hbar} x_{0}^{2}\right) .
$$

\section{DIPOLE PUMP}

First, we consider the time evolution of a particle with the initial wave function in the potential

$$
V(x, t)=\frac{1}{2} m \omega^{2}[x-U(t)]^{2}
$$

where $U(t)$ is some square integrable function. This kind of potential is a generalization of the parabolic potential with $U(t)=0$ and $V(x, t)=\frac{1}{2} m \omega^{2} x^{2}$ used, in particular, in the analysis of the movement of channeling charged particles [6, 7]. In this case, for the wave function $\Psi=\Psi\left(x, t ; x_{0}, t_{0}\right)$, the Schrödinger equation has the form [5]

$$
\frac{\hbar}{i} \frac{\partial \Psi}{\partial t}=-\frac{\hbar^{2}}{2 m} \frac{\partial^{2} \Psi}{\partial x^{2}}+\frac{1}{2} m \omega^{2}[x-U(t)]^{2} \Psi
$$

The physical content of the wave function $\Psi$ is that it describes the amplitude of the probability of transition from state to moment $t_{0}=0$, characterized by a coordinate $x_{0}$, to a state at a moment $t$, characterized by a coordinate $x$. Based on the parabolicity of the potential (2), we will look for a solution to equation (3) in the form

$$
\Psi\left(x, t ; x_{0}, t_{0}\right)=\exp \left(C_{0}(t)+C_{1}(t) x+C_{2}(t) x^{2}\right),
$$

with some functions $C_{0}(t), C_{1}(t), C_{2}(t)$. For them, one can compose a system of three ordinary differential equations with initial conditions. As a result, we find that the solution of the Schrödinger equation (3) is as follows [8]

$$
\begin{gathered}
\Psi\left(x, t ; x_{0}, t_{0}\right)=\left(\frac{m e^{i \omega t}}{\pi \hbar\left(e^{2 i \omega t}-1\right)}\right)^{1 / 2} \times \\
\times \exp \left(-\frac{m \omega}{2 \hbar}\left(x^{2}-x_{0}^{2}\right)-i \frac{m \omega^{2}}{2 \hbar} \int_{0}^{t} U^{2}(\tau) d \tau\right) \times \\
\times \exp \left(i \frac{\hbar}{2 m} \int_{0}^{t} Y^{2}(\tau) d \tau+x Y(t)-\right. \\
\left.-\frac{m \omega}{\hbar\left(e^{2 i \omega t}-1\right)}\left[x-e^{i \omega t} x_{0}-R(t)\right]^{2}\right),
\end{gathered}
$$

where

$$
\begin{gathered}
Y(\tau)=i \frac{m \omega^{2}}{\hbar} \int_{0}^{\tau} U\left(\tau^{\prime}\right) \exp \left(-i \omega \tau+i \omega \tau^{\prime}\right) d \tau^{\prime}, \\
R(t)=\frac{\hbar e^{i \omega t}}{m} \int_{0}^{t} Y(\tau) \exp (-i \omega \tau) d \tau .
\end{gathered}
$$

The Gaussian form of expression (4) for the obtained function $\Psi\left(x, t ; x_{0}, t_{0}\right)$ (the Green function) is a consequence of the parabolicity of potential (2). Function $\Psi\left(x, t ; x_{0}, t_{0}\right)$ (4) satisfies the same equation as the wave function $\psi\left(x_{0}, 0\right)$, but with the initial condition $\Psi\left(x, t ; x_{0}, 0\right)=\delta\left(x-x_{0}\right)$ 
The value of the wave function $\psi(x, t)$ at the time $t>t_{0}$ can be determined by its given value $\psi\left(x_{0}, 0\right)$ at the moment $t_{0}=0$ :

$$
\psi(x, t)=\int_{-\infty}^{\infty} \psi\left(x_{0}, 0\right) \Psi\left(x, t ; x_{0}, 0\right) d x_{0} .
$$

After calculating the integral (5) with the core $\Psi\left(x, t ; x_{0}, 0\right)(4)$ and the initial wave function $\psi\left(x_{0}, 0\right)$ (1), we obtain

$$
\begin{aligned}
& \psi(x, t)=\left(\frac{m \omega}{\pi \hbar}\right)^{1 / 4} \exp \left(-i \frac{m \omega^{2}}{2 \hbar} J-\frac{m \omega}{2 \hbar}[x-X(t)]^{2}\right), \\
& X(t)=\omega \int_{0}^{t} U(\tau) \sin (\omega t-\omega \tau) d \tau,
\end{aligned}
$$

where $J$ is the phase incursion of the resulting wave function

$$
\begin{aligned}
& J=\int_{0}^{t} U^{2}(\tau) d \tau+\frac{t \hbar}{m \omega}+2 x \int_{0}^{t} U(\tau) \cos (\omega(t-\tau)) d \tau+ \\
& +\int_{0}^{t} d \tau \int_{0}^{t} d \tau^{\prime} U(\tau) U\left(\tau^{\prime}\right) \sin (\omega(\tau-t)) \cos \left(\omega\left(\tau^{\prime}-t\right)\right) .
\end{aligned}
$$

If the particle at $t=0$ was in the ground state with the wave function (1), then by the time $t$ moment for the probability density $p(x, t)$ we find

$$
p(x, t)=|\psi(x, t)|^{2}=\left(\frac{m \omega}{\pi \hbar}\right)^{1 / 2} \exp \left(-\frac{m \omega}{\hbar}[x-X(t)]^{2}\right)
$$

Thus, if a particle experiences a temporal evolution in potential (2) with $U(\tau)=0$, then by the time instant $t$ the distribution of probabilities $p(x, t)$ remains the same as at the moment $t_{0}=0$. The behavior of the wave packet changes significantly if $U(\tau) \neq 0$ that can be seen from the characteristics of the wave packet.

Quantum-mechanical averages - the first and second moments of the density of the probability distribution $p(x, t)$

$$
\begin{gathered}
\langle x(t)\rangle=\omega \int_{0}^{t} U(\tau) \sin (\omega t-\omega \tau) d \tau, \\
\left\langle x^{2}(t)\right\rangle=\frac{\hbar}{2 m \omega}+\omega^{2} \times \\
\times \int_{0}^{t} \int_{0}^{t} U(\tau) U\left(\tau^{\prime}\right) \sin (\omega t-\omega \tau) \sin \left(\omega t-\omega \tau^{\prime}\right) d \tau d \tau^{\prime} \\
\text { experiencing swing. }
\end{gathered}
$$

If the modulation function $U(t)$ contains a stochastic component, then $\langle x(t)\rangle$ and $\left\langle x^{2}(t)\right\rangle$ are also timedependent random variables.

Next we will consider the case of parametric resonance, when

$$
U(t)=g \sin (\omega t),
$$

where $g$ is the modulation index, rather small compared to 1 . In this case, we have

$$
\begin{gathered}
\langle x(t)\rangle=\frac{1}{2} g \omega t\left(\cos (\omega t)-\frac{\sin (\omega t)}{\omega t}\right) \\
\left\langle x^{2}(t)\right\rangle=\frac{\hbar}{2 m \omega}+\frac{1}{4} g^{2} \omega^{2} t^{2}\left(\cos (\omega t)-\frac{\sin (\omega t)}{\omega t}\right)^{2}, \\
\langle p(t)\rangle=\frac{1}{2} g \omega^{2} t \sin (\omega t) \\
\left\langle p^{2}(t)\right\rangle=\frac{\hbar \omega}{2 m}+\frac{1}{4}\left[g m \omega^{2} \sin (\omega t)\right]^{2}
\end{gathered}
$$

As a result, we obtain for the average energy

$$
\begin{gathered}
E=\frac{1}{2 m}\left\langle p^{2}\right\rangle+\frac{m}{2} \omega^{2}\left\langle x^{2}\right\rangle= \\
=\frac{\hbar \omega}{2}+\frac{g^{2} \hbar \omega}{2}\left(\omega^{2} t^{2}+\omega t \sin (2 \omega t)+\sin ^{2}(\omega t)\right) .
\end{gathered}
$$

The rate of increase in the average particle energy over time is

$$
r_{t}=\frac{E}{E_{0}}=1+g^{2}\left(\omega^{2} t^{2}+\omega t \sin (2 \omega t)+\sin ^{2}(\omega t)\right) \text {. }
$$

The potential $V(x, t)$ with the buildup of the form (11) on the interval $0 \leq t \leq 50$ is shown in Fig. 1 .

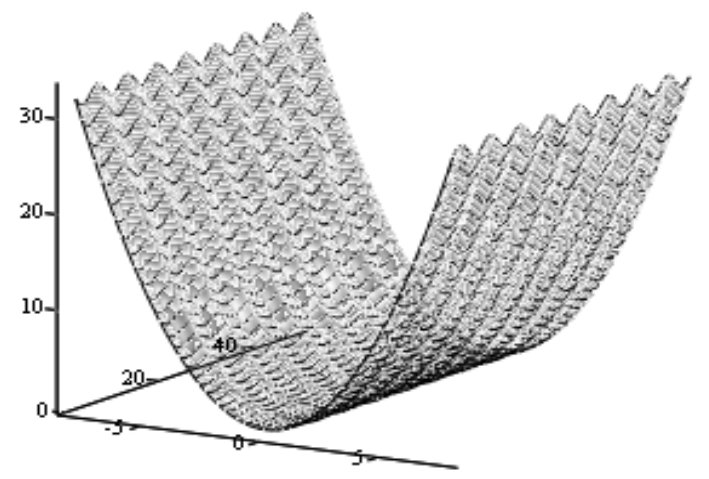

Fig. 1. Potential $V(x, t) ; \omega=1, g=0.1$

The temporal evolution of the probability distribution density over the interval $0 \leq t \leq 50$ is shown in Fig. 2.

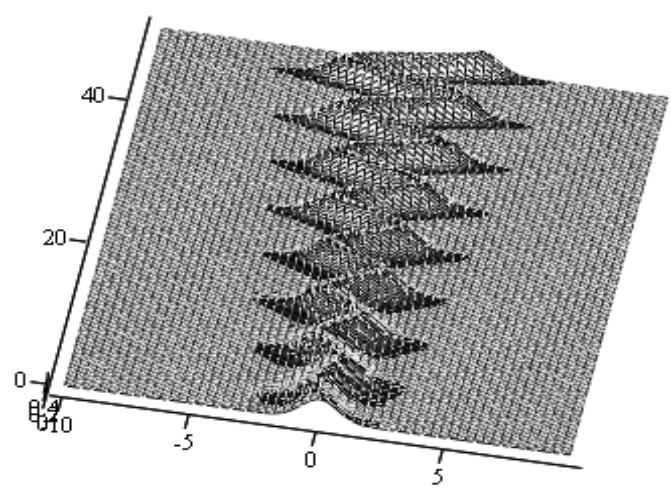

Fig. 2. The density of probability distribution $p(x, t)$ in the interval $-8 \leq x \leq 8 ; \omega=1, g=0.1$

As a result of the resonant buildup, the average energy of a particle increases with time according to a parabolic law (Fig. 3). 


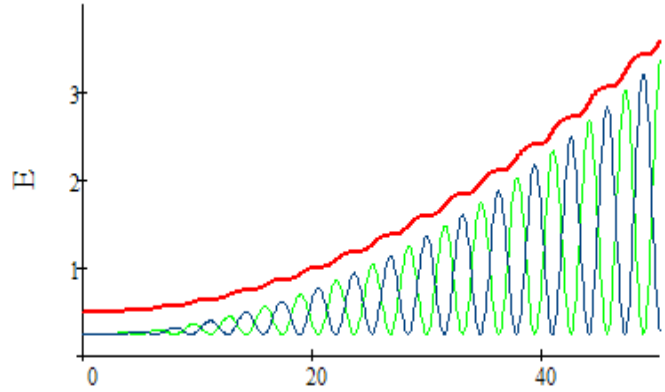

$\mathrm{t}$

Fig. 3. The dependence of the average energy $E$ (red line) on the current time $t, \omega=1, g=0.1$. The blue line is kinetic energy, green line - potential energy

\section{QUADRUPOLE PUMP}

The problem of the dynamics of the wave function in a parabolic potential subject to periodic perturbations was considered in [10]. In the present work, a more detailed study of the wave packet dynamics has been carried out. Below, we will consider the time evolution of a particle with the initial wave function

$$
\psi\left(x_{0}, 0\right)=\left(\frac{m \omega}{\pi \hbar}\right)^{1 / 4} \exp \left(-\frac{m \Omega(0)}{2 \hbar} x_{0}^{2}\right)
$$

in potential $V\left(x_{t}, t\right)=m \Omega^{2}(t) x_{t}^{2} / 2$, where $\Omega(t)$ is some given function. This kind of potential is a generalization of the parabolic potential $V(x, t)=m \omega^{2} x^{2} / 2$ with a constant frequency $\omega$. In this case, the Schrödinger equation for the Green function $\Psi=\Psi\left(x_{t}, t ; x_{0}, t_{0}\right)$ is

$$
\frac{\hbar}{i} \frac{\partial}{\partial t} \Psi=-\frac{\hbar^{2}}{2 m} \frac{\partial^{2}}{\partial x_{t}^{2}} \Psi+\frac{1}{2} m \Omega^{2}(t) x_{t}^{2} \Psi .
$$

The paper will consider the case of parametric resonance, when

$$
\Omega^{2}(t)=\omega^{2}(1+g \sin (2 \omega t)),
$$

where $\omega$ is the natural frequency, $g$ is the modulation index, rather small compared to 1 .

In the case when the frequency is constant, $\Omega(t)=$ cons , for equation (19) it is possible to obtain analytical expressions in quadratures. In the general case of a frequency-dependent frequency $\Omega(t)$ for calculating the dynamics of quantum-mechanical wave functions in a perturbed potential, a recurrent algorithm can be constructed. This is based on the fact that for any $t$ wave function $\psi\left(x_{t}, t ; x_{0}, t_{0}\right)$ has a normal appearance with respect to $x_{t}$ and $x_{0}$. Therefore, it is sufficient to construct an algorithm for calculating the first two of its moments. We use the explicit form of solving the Schrödinger equation with a constant frequency $\omega$ :

$$
\begin{gathered}
\Psi\left(x_{t}, t ; x_{0}, t_{0}\right)=\left(\frac{m e^{i \omega t}}{\pi \hbar\left(e^{2 i \omega t}-1\right)}\right)^{1 / 2} \times \\
\exp \left(-\frac{m \omega}{2 \hbar}\left(x_{t}^{2}-x_{0}^{2}\right)-\frac{m \omega}{\hbar\left(e^{2 i \omega t}-1\right)}\left(x_{t}-e^{i \omega t} x_{0}\right)^{2}\right)
\end{gathered}
$$

and rewrite it for two points in time $t_{k-1}$ and $t_{k}$ separated by a sufficiently small interval $\tau$ :

$$
\begin{gathered}
\Psi\left(x_{k}, t_{k} ; x_{k-1}, t_{k-1}\right)=\left(\frac{m q_{k}}{\pi \hbar\left(q_{l}^{2}-1\right)}\right)^{1 / 2} \times \\
\exp \left(-\frac{m \Omega_{k}}{2 \hbar}\left(x_{k}^{2}-x_{k-1}^{2}\right)-\frac{m \Omega_{k}}{\hbar\left(q_{k}^{2}-1\right)}\left(x_{k}-q_{k} x_{k-1}\right)^{2}\right),
\end{gathered}
$$

where $q_{k}=\exp \left(i \Omega_{k} \tau\right), \Omega_{k}=\Omega\left(t_{k}\right)$ - the frequency values at each of the time intervals.

The value of the wave function $\psi\left(x_{k}, t_{k}\right)$ at the time $t_{k}>t_{0}$ can be determined by the value given at the time $t_{k-1}$ of its value $\psi\left(x_{k-1}, t_{k-1}\right)$ in accordance with the ratio

$$
\psi\left(x_{k}, t_{k}\right)=\int_{-\infty}^{\infty} \psi\left(x_{k-1}, t_{k-1}\right) \Psi\left(x_{k}, t_{k} ; x_{k-1}, t_{k-1}\right) d x_{k-1} \text {. }
$$

Whence it is clear that one can use the property of normality and limit oneself to the first two points. Since $\left\langle x_{k}\right\rangle=0$ for all $k$,

$$
\begin{aligned}
& \psi\left(x_{k}, t_{k}\right)=N \int_{-\infty}^{\infty} \exp \left(-Q\left(x_{k-1}, x_{k}\right)\right) d x_{k-1}, \\
& Q\left(x_{k-1}, x_{k}\right)=\frac{m \Omega_{k}}{\hbar\left(q_{k}^{2}-1\right)}\left(x_{k}-q_{k} x_{k-1}\right)^{2}+ \\
& +\frac{1}{2 s_{k-1}^{2}} x_{k-1}^{2}+\frac{m \Omega_{k}}{2 \hbar}\left(x_{k}^{2}-x_{k-1}^{2}\right),
\end{aligned}
$$

where $N$ is the normalization, $s_{k-1}^{2}$ is the dispersion of the wave function at the moment $t_{k-1}$.

Our goal is to construct a recurrent relation connecting the dispersions $s_{k-1}^{2}$ and $s_{k}^{2}$ of the wave function for subsequent moments of time. Given that $\left\langle x_{k-1}\right\rangle=\left\langle x_{k}\right\rangle=0$, we get $s_{k-1}^{2}=\left\langle x_{k-1}^{2}\right\rangle$ and $s_{k}^{2}=\left\langle x_{k}^{2}\right\rangle$. As a result of integration, we arrive at the following recurrent expression relating the current variance $s_{k}^{2}$ with the previous variance $s_{k-1}^{2}$ :

$$
\begin{gathered}
s_{k}^{2}=\frac{1}{\eta_{k}} \frac{\sin \left(\Omega_{k} \tau\right)+i \eta_{k} s_{k-1}^{2} \cos \left(\Omega_{k} \tau\right)}{i \cos \left(\Omega_{k} \tau\right)+\eta_{k} s_{k-1}^{2} \sin \left(\Omega_{k} \tau\right)}, \\
\eta_{k}=\frac{m}{\hbar} \Omega_{k} \equiv \frac{m}{\hbar} \Omega\left(t_{k}\right) .
\end{gathered}
$$

Next, we will apply the rule of frequency modulation $\Omega(t)$ at a potential with time according to relation (20). Denoting $\xi_{k}=\eta_{k+1} s_{k}^{2}$, we construct a difference expression on the basis of (23)

$$
\xi_{k}-\xi_{k-1}=\frac{\Omega_{k} \tau\left(1-\xi_{k-1}^{2}\right)}{i}+\frac{\Omega_{k+1}-\Omega_{k}}{\Omega_{k}} \xi_{k-1}+o(\tau) \text {. }
$$

Since at small modulation index $g$ we have $\left(\Omega_{k+1}-\Omega_{k}\right) \Omega_{k+1}^{-1}=g \omega \tau \cos \left(2 \omega t_{k}\right)+O\left(g^{2}\right)$, then going to the limit $\tau \rightarrow 0$, getting

$$
\frac{d \xi}{d t}=-i \Omega(t)\left(1-\xi^{2}\right)+g \omega \xi \cos (2 \omega t),
$$


To solve equation (26), we use the substitution $\xi=-i \operatorname{tg}(\mu)$. This gives

$$
\frac{d \mu}{d t}=\Omega(t)+\frac{1}{2} g \omega \cos (2 \omega t) \sin (2 \mu) .
$$

On the right of this equation, we select the resonant term, and reject the nonresonant term,

$$
\frac{d}{d t}\left(2 \mu-2 \int_{0}^{t} \Omega\left(t^{\prime}\right) d t^{\prime}\right)=\frac{1}{2} g \omega \sin (2 \mu-2 \omega t),
$$

then for the function $Z=\mu-\int_{0}^{t} \Omega\left(t^{\prime}\right) d t^{\prime}+\omega t$ we get the equation

$$
\frac{d}{d t}(2 Z)=\frac{1}{2} g \omega \sin \left(2 Z+2 \int_{0}^{t} \Omega\left(t^{\prime}\right) d t^{\prime}-2 \omega t\right) .
$$

Under the sine sign, the second and third terms have order $O(g)$. Taking into account the smallness of the modulation index $g$, we find the first integral of the equation (26)

$$
\operatorname{tg} \mu=\frac{\operatorname{tg} \varphi+W \operatorname{tg}\left(\mu_{0}\right)}{1-W \operatorname{tg}\left(\mu_{0}\right) \operatorname{tg} \varphi}
$$

where

$$
W=\exp \left(\frac{g \omega t}{2}+g^{2} \sin ^{2} \omega t\right), \varphi=\omega t+\frac{g}{2} \sin ^{2} \omega t
$$

From here follows the desired dependence for the mean-square size of the wave function:

$$
s^{2}(t)=\frac{1}{\eta(t)} \frac{s^{2}(0) W-i \operatorname{tg} \varphi}{1-i s^{2}(0) W \operatorname{tg} \varphi} .
$$

Let us assume that at the initial moment the particle is in the ground state with $s^{2}(0)=1$. Based on expression (31) for the dispersion $s^{2}(t)$ of the wave packet and the normality property, one can find the necessary characteristics of the wave function.

The size of the wave packet in the coordinate and momentum:

$$
\begin{aligned}
& \left\langle x^{2}\right\rangle=\frac{1}{2 \eta(t) W}\left(W^{2} \cos ^{2} \varphi+\sin ^{2} \varphi\right), \\
& \left\langle p^{2}\right\rangle=\frac{\hbar^{2} \eta(t)}{2 W}\left(W^{2} \sin ^{2} \varphi+\cos ^{2} \varphi\right) .
\end{aligned}
$$

Over time, the average energy is

$$
E=\frac{1}{2 m}\left\langle p^{2}\right\rangle+\frac{m}{2} \Omega_{t}^{2}\left\langle x^{2}\right\rangle=\frac{\hbar \Omega(t)}{2} \operatorname{ch}\left(\frac{g \omega t}{2}+g^{2} \sin ^{2} \omega t\right) .
$$

The rate of increase in the average particle energy over time is as follows

$$
r_{t}=\frac{E}{E_{0}}=\sqrt{1+g \sin (2 \omega t)} \operatorname{ch}\left(\frac{g \omega t}{2}+g^{2} \sin ^{2} \omega t\right) \text {. }
$$

The expressions found allow for a known function of frequency $\Omega(t)$ and a given dispersion $s_{0}^{2}$ of the initial wave function $\psi\left(x_{0}, 0\right)$ to determine the sequence of sizes $s_{t}^{2}$ of wave functions $\psi\left(x_{t}, t\right)$ for an arbitrary number of periods of compelling oscillations. Within each of their periods, the size of the wave function at the coordinate undergoes oscillations according to (30), defined $\omega$ and $g$. Since the wave function $\psi\left(x_{t}, t\right)$ has a normal form with zero mean and variance $s^{2}(t)$, the particle state is thereby completely de- termined. The average energy of a particle experiences growth according to the law of hyperbolic cosine, which, in contrast to [10], additionally still experiences modulation with frequency $2 \omega$.

The potential $V(x, t)$ with the buildup of the form (20) on the interval $0 \leq t \leq 50$ is shown in Fig. 4 .

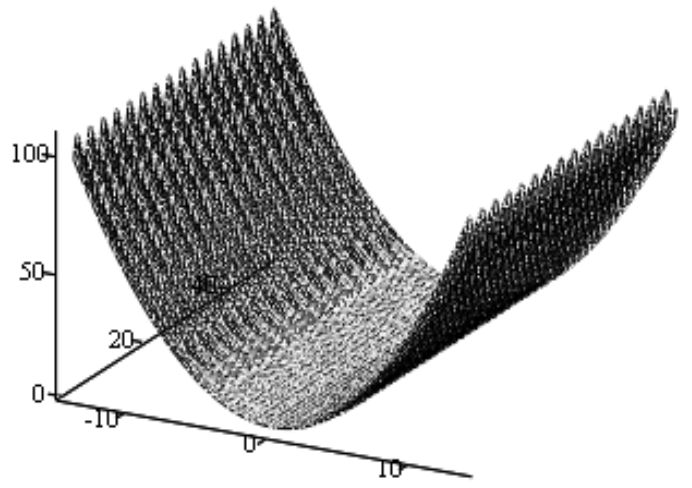

Fig. 4. Potential $V(x, t) ; \omega=1, g=0.1$

The time evolution of the probability distribution density $p(x, t)=|\psi(x, t)|^{2}$ over the interval $0 \leq t \leq 50$ for the values of the parameters $\omega=1$ and $g=0.1$ is shown in Fig. 5.

In Fig. 6 shows the dependence of the average energy $E$ on the current time $t$ at $\omega=1$ and $g=0.1$. It is seen that the average energy $E$ increases, while experiencing a modulation with a frequency of $2 \omega$. The amplitude of this modulation, in turn, is determined by the index value $g$.

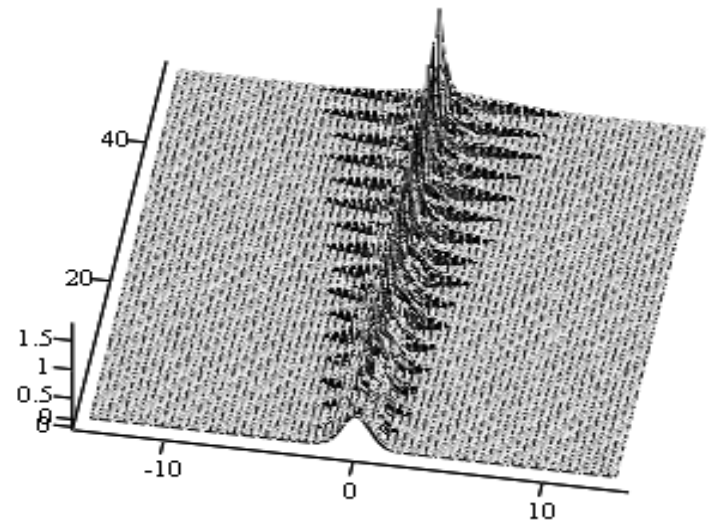

Fig. 5. Probability distribution density $p(x, t)$ on the interval $-14 \leq x \leq 14 ; \omega=1, g=0.1$

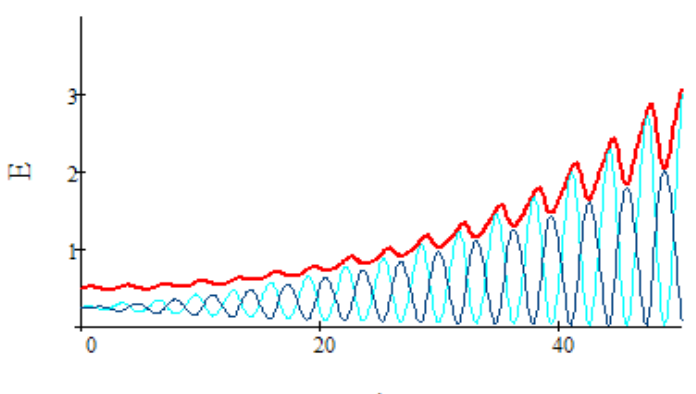

Fig. 6. The dependence of the total energy $E$ (red line) on the current time $t ; \omega=1, g=0.1$. The blue line is kinetic energy, green line-potential energy 


\section{CONCLUSIONS}

We consider the parabolic potential, which as a whole is subject to a dipole or quadrupole effect (parametric resonance), which varies periodically with time, and the dynamics of the wave function of a particle in it. Based on the nonstationary Schrödinger equation, analytical expressions and algorithms for calculating the dynamics of the wave function are constructed. The evolution of the particle wave function is analyzed. The asymptotic equations of motion are given, with the help of which the main characteristics of the wave packet are obtained. For selected types of dipole or quadrupole perturbations of the potential, examples of the evolution of the wave function and the average particle energy are given. Note that the developed approach can be extended to the case of a stochastic modulation perturbation.

\section{REFERENCES}

1. V.I. Dubinko, D.V. Laptev. Chemical and nuclear catalysis driven by localized anharmonic vibration // Letter on materials. 2016, № 6(1), p. 16-21.

2. V.I. Dubinko, D.V. Laptev, D. Terentyev, S. Dmitriev, K. Irwin. Assessment of discrete breathers in the metallic hydrides // Computation Material Science. 2019, v. 158, p. 389-397.

3. V.I. Dubinko, D.V. Laptev, A.S. Mazmanishvili, J.F.R. Archilla. Quantum dynamics of wave packets in a nonstationary parabolic potential and the Kramers escape rate theory // Journal of Micromechanics and Molecular Physics. 2016, v. 01, № 02, p. 1650010 .

4. A.S. Mazmanishvili, I.A. Knyaz'. Dynamics of quantum particles in perturbed parabolic $2 \mathrm{~d}$ potential // Journal of Nano- and Electronic Physics. 2016, v. 8, № 4, p. 04014(6cc).

5. A.A. Sokolov, Yu.I. Loskutov, I.M. Ternov. Quantum mechanics. M.: "Science", 1962, 591 p.

6. A.I. Akhiezer, N.F. Shulga. Electrodynamic of high energies in matter. M.: "Science", 1993, 344 p.

7. V.G. Baryshevsky. Channeling, radiation and reactions in crystals at high energies. Minsk: "BSU Publishing House", 1982, $256 \mathrm{p}$.

8. A.S. Mazmanishvili. Path integration as a role for solving physical problems. K.: "Naukova Dumka", 1987, $224 \mathrm{p}$.

9. V.I. Dubinko, P.A. Selyshchev, J.F.R. Archilla. Reaction rate theory with crystal anharmonicity // Phys. Rev. 2011, v. 83 (4), p. 041124-13.

10. I.I. Goldman, V.D. Krivchenkov. Collection of problems in quantum mechanics. M.: "Gosizdat of technical and theoretical literature", 1957, $275 \mathrm{p}$.

Article received 05.04.2019

\section{РЕЗОНАНСНАЯ РАСКАЧКА ВОЛНОВОЙ ФУНКЦИИ В ПАРАБОЛИЧЕСКОМ ПОТЕНЦИАЛЕ}

\section{А.С. Мазманишвили}

Рассмотрен параболический потенциал, который как целое подвержен дипольному или квадрупольному воздействию (параметрическому резонансу), периодически меняющемуся с течением времени, и динамика волновой функции частицы в нем. На основе найденных решений нестационарного уравнения Шредингера построены алгоритмы расчета динамики волновой функции. Проанализирована эволюция волновой функции частицы. Приведены асимптотические решения уравнения движения, с помощью которых получены основные характеристики волнового пакета. Для выбранных видов возмущения потенциала приведены примеры эволюции волновой функции.

\section{РЕЗОНАНСНЕ РОЗГОЙДУВАННЯ ХВИЛЬОВОЇ ФУНКЦЇ̈ В ПАРАБОЛІЧНОМУ ПОТЕНЦАЛІ О.С. Мазманішвілі}

Розглянуто параболічний потенціал, який як ціле схильний дипольному або квадрупольному впливу (параметричному резонансу), що періодично змінюється з плином часу, і динаміка хвильової функції частинки в ньому. На основі отриманих розв'язків нестаціонарного рівняння Шредінгера побудовані алгоритми розрахунку динаміки хвильової функції. Проаналізовано еволюцію хвильової функції частинки. Наведено асимптотичні рішення рівняння руху, за допомогою яких отримані основні характеристики хвильового пакета. Для обраних видів збурення потенціалу наведені приклади еволюції хвильової функції. 